\title{
On the accretion luminosity of isolated neutron stars
}

\author{
N. R. Ikhsanov^ \\ Max-Planck-Institut für Radioastronomie, Auf dem Hügel 69, 53121 Bonn, Germany \\ Central Astronomical Observatory of the Russian Academy of Sciences, Pulkovo 65/1, 196140 St. Petersburg, Russia \\ Isaac Newton Institute of Chile, St. Petersburg Branch, Russia
}

Received 14 October 2002 / Accepted 6 December 2002

\begin{abstract}
The accretion process onto a magnetized isolated neutron star, which captures material from the interstellar medium, is discussed. The evolutionary track of such a star can be presented as a sequence of four states: ejector, supersonic propeller, subsonic propeller, and steady accretor. I show that subsonic propeller $\rightarrow$ accretor transition does not occur as long as the magnetic field of the star is strong enough to control the accretion flow in the stellar vicinity. During the subsonic propeller state the accretion rate onto the stellar surface is limited to the rate of plasma diffusion into its magnetosphere. The diffusion rate is at least three orders of magnitude smaller than the maximum possible mass capture rate by the star. Therefore, the expected accretion luminosity of magnetized isolated neutron stars is at least three orders of magnitude smaller than that previously evaluated.
\end{abstract}

Key words. accretion, accretion disks - magnetic fields - stars: neutron - X-rays: stars

\section{Introduction}

The observational appearance of isolated neutron stars (INSs), which accrete material from the interstellar medium, is a subject of intensive theoretical investigation (e.g. Shvartsman 1970; Treves \& Colpi 1991; Treves et al. 2000; Toropina et al. 2001; Popov et al. 2000a). It is presently established that the initially fast rotating INSs, under certain conditions, are able to switch their state from ejector to propeller within the Hubble time $t<t_{\mathrm{H}} \approx 10^{10} \mathrm{yr}$, and even to reach the periods at which the corotational radius,

$R_{\text {cor }}=\left(G M_{\mathrm{ns}} / \omega^{2}\right)^{1 / 3}$,

exceeds the magnetospheric radius of the star,

$R_{\mathrm{m}} \equiv\left(\mu^{2} / \dot{M}_{\mathrm{c}} \sqrt{2 G M_{\mathrm{ns}}}\right)^{2 / 7}$.

Here $M_{\mathrm{ns}}, \omega=2 \pi / P_{\mathrm{s}}$ and $\mu$ are the mass, the angular velocity, and the dipole magnetic moment of the neutron star, respectively. $\dot{M}_{\mathrm{c}}$ is the mass capture rate by the star from the interstellar medium, which can be expressed as

$\dot{M}_{\mathrm{c}} \simeq \pi R_{\alpha}^{2} \rho V_{\infty}$

$V_{\infty}$ is the relative velocity of the neutron star to the surrounding material, $\rho$ is the density of the interstellar material, and $R_{\alpha}$ is the accretion radius of the star,

$R_{\alpha}=2 G M_{\mathrm{ns}} / V_{\infty}^{2}$.

\footnotetext{
* e-mail: ikhsanov@mpifr-bonn.mpg.de
}

Limiting $\rho<10^{-24} \mathrm{~g} \mathrm{~cm}^{-3}$ and $V_{\infty}>V_{\mathrm{s}}$, where $V_{\mathrm{s}}$ is the thermal velocity in the interstellar material, Popov et al. (2000a) have estimated the maximum possible rate of mass capture by an isolated neutron star as

$\dot{M}_{\mathrm{c}} \lesssim \dot{M}_{\max } \simeq 10^{12} \rho_{-24} m^{2} V_{6}^{-3} \mathrm{~g} \mathrm{~s}^{-1}$.

Here $m$ is the mass of the star expressed in units of $M_{\odot}, \rho_{-24}=$ $\rho / 10^{-24} \mathrm{~g} \mathrm{~cm}^{-3}$ and $V_{6}=V_{\infty} / 10^{6} \mathrm{~cm} \mathrm{~s}^{-1}$. Then, assuming that all material captured by the star is accreted onto its surface, they have suggested that the accretion luminosity of INSs can be as high as $\dot{M}_{\max } G M_{\mathrm{ns}} / R_{\mathrm{ns}} \sim 10^{32} \mathrm{erg} \mathrm{s}^{-1}$.

The analysis of the validity of this assumption is the subject of the present paper. I show that under the conditions of interest the mass accretion rate onto the stellar surface is limited by the rate of plasma penetration into the stellar magnetosphere, which is significantly smaller than $\dot{M}_{\max }$. Therefore, the maximum possible accretion luminosity of a magnetized isolated neutron star proves to be limited to a few $\times 10^{28} \mathrm{erg} \mathrm{s}^{-1}$. The evolutionary tracks of isolated neutron stars are briefly reviewed in the next section. The rate of plasma penetration into the magnetosphere and, correspondingly, the mass accretion rate onto the surface of isolated neutron stars are evaluated in Sect. 3. Here, I also show that the rate of mass accretion onto the surface of an isolated neutron star remains smaller than $\dot{M}_{\mathrm{c}}$ as long as $R_{\mathrm{m}} \gg R_{\mathrm{ns}}$. The main conclusions are summarized in Sect. 4.

\section{Evolutionary tracks of INSs}

As was first recognized by Shvartsman (1970), the evolutionary track of a rotating magnetized neutron star can be presented in 
the form of the following sequence of its states: ejector $\rightarrow$ propeller $\rightarrow$ accretor. Within this scheme, the rotational rate of a newly born fast rotating neutron star decreases, initially by the generation of the magneto-dipole waves and ejection of relativistic particles (pulsar-like spin-down), and later by means of the interaction between its magnetosphere and the surrounding material (propeller spin-down). The first state transition occurs when the pressure of the material ejected by the star can no longer balance the pressure of the surrounding gas, and the latter, penetrating into the accretion radius of the star, interacts with the stellar magnetosphere. A detailed analysis of this state transition with respect to INSs was presented by Popov et al. (2000b).

The spin evolution of a spherically accreting strongly magnetized neutron star in the state of propeller has been investigated by Davies et al. (1979) and Davies \& Pringle (1981). As they shown, two sub-states of the propeller state can be distinguished: the supersonic and subsonic propeller. In both cases the neutron star is spinning down due to the interaction between its magnetosphere and the surrounding gas. As a result of this interaction, the star's magnetosphere is surrounded by a spherical quasi-static atmosphere, in which the plasma temperature is of the order of the free-fall temperature

$T_{\mathrm{ff}}(r)=\left(G M_{\mathrm{ns}} m_{\mathrm{p}}\right) /(k r)$.

Here $G, m_{\mathrm{p}}$, and $k$ are the gravitational constant, the proton mass, and the Boltzmann constant, respectively.

The atmosphere is extended from the magnetospheric boundary up to the accretion radius of the neutron star. The rotational energy loss by the neutron star is convected up through the atmosphere by the turbulent motion and lost through its outer boundary.

The formation of the atmosphere in the first approximation prevents the surrounding gas from penetrating to within the accretion radius of the star. As the neutron star moves through the interstellar medium, the interstellar gas overflows the outer edge of the atmosphere with the rate $\dot{M}_{\mathrm{c}}$ (see Eq. (2)), which is traditionally called the strength of the stellar wind and denotes the maximum possible mass capture rate by the neutron star.

\subsection{Supersonic propeller}

As long as the angular velocity of the neutron star is large enough for the corotational radius to be smaller than the magnetospheric radius, the star is in the centrifugal inhibition regime (i.e. the centrifugal acceleration at the magnetospheric boundary, $\omega^{2} R_{\mathrm{m}}$, dominates the gravitational acceleration, $G M_{\mathrm{ns}} / R_{\mathrm{m}}^{2}$ ). The centrifugal inhibition is not effective only within the bases of the corotational cylinder. However, the accretion of material onto the stellar surface through these regions does occur only if the the angle between the magnetic and rotational axes is small enough (see Ikhsanov 2001c) and if the magnetic field of the star is weak enough for the magnetospheric radius to exceed the stellar radius only by a factor of 2-3 (for discussion see Toropin et al. 1999; Romanova et al. 2002). Otherwise, the accretion power is significantly smaller than the spin-down power due to propeller action by the fast rotating star.
Except the bases of the corotational cylinder, the linear velocity at the boundary of the magnetosphere, which is corotating with the star, in this case is larger than the sound speed in the atmospheric plasma. That is why this state is usually refereed to as a supersonic propeller (see also Ikhsanov 2002).

\subsection{Subsonic propeller}

As the star is spinning down, its corotational radius increases and reaches the magnetospheric radius when $P_{\mathrm{s}}=P_{\mathrm{cd}}$, where

$P_{\mathrm{cd}} \simeq 23 \mu_{30}^{6 / 7} \mathrm{~m}^{-5 / 7} \dot{M}_{15}^{-3 / 7} \mathrm{~s}$.

Here $\dot{M}_{15}$ is the strength of the stellar wind expressed in units of $10^{15} \mathrm{~g} \mathrm{~s}^{-1}$, and $\mu_{30}$ is the dipole magnetic moment of a neutron star expressed in units of $10^{30} \mathrm{G} \mathrm{cm}^{3}$.

Under the condition $P_{\mathrm{s}}>P_{\mathrm{cd}}$ the centrifugal barrier is not effective: the atmospheric plasma, penetrating into the magnetic field of the star, is able to flow along the magnetic field lines and to accrete onto the stellar surface. However, as shown by Arons \& Lea (1976) and Elsner \& Lamb (1976), the rate of plasma penetration into the magnetosphere of a spherically accreting strongly magnetized neutron star can be as high as $\dot{M}_{\mathrm{c}}$ only if the magnetospheric boundary is unstable with respect to interchange (e.g. Rayleigh-Taylor) instabilities. Otherwise, the rate of plasma penetration is limited to the diffusion rate, which is a few orders of magnitude smaller than $\dot{M}_{\mathrm{c}}$ (see Eq. (10)). For instability to occur the sign of the effective gravitational acceleration at the magnetospheric boundary should be positive:

$g_{\text {eff }}=\frac{G M_{\mathrm{ns}}}{R_{\mathrm{m}}^{2}(\theta)} \cos \theta-\frac{V_{\mathrm{T}_{\mathrm{i}}}^{2}\left(R_{\mathrm{m}}\right)}{R_{\text {curv }}(\theta)}>0$.

Here $R_{\text {curv }}$ is the curvature radius of the field lines, $\theta$ is the angle between the radius vector and the outward normal to the magnetospheric boundary and $V_{\mathrm{T}_{\mathrm{i}}}\left(R_{\mathrm{m}}\right)$ is the ion thermal velocity of the accreting plasma at the boundary. For the case of the equilibrium magnetospheric shape derived by Arons \& Lea (1976), the condition (7) can be expressed in terms of the plasma temperature at the magnetospheric boundary as

$T<T_{\text {cr }} \simeq 0.3 T_{\text {ff }}$.

This indicates that the condition $R_{\mathrm{m}}<R_{\mathrm{cor}}$ is necessary, but not sufficient for the effective accretion (with the rate of $\sim \dot{M}_{\mathrm{c}}$ ) onto the surface of neutron star to start. In addition, it is required that the cooling of plasma at the base of the atmosphere is more effective than the heating.

As shown by Davies \& Pringle (1981), the cooling of the atmospheric plasma is governed by the bremsstrahlung radiation and the convective motion. For these processes to dominate the energy input into the atmosphere due to the propeller action by the star, the spin period of the star should be $P_{\mathrm{s}} \gtrsim P_{\mathrm{br}}$, where $P_{\mathrm{br}}$ is a so-called break period, which according to Ikhsanov (2001a) is

$P_{\mathrm{br}} \simeq 450 \mu_{30}^{16 / 21} \dot{M}_{15}^{-5 / 7} \mathrm{~m}^{-4 / 21} \mathrm{~s}$.

Under the conditions of interest, the break period significantly exceeds $P_{\mathrm{cd}}$. This means that the state transition supersonic propeller $\rightarrow$ steady accretor may occur only via an additional 
intermediate state, which is called the subsonic propeller. This term reflects the fact that the rotation velocity of the magnetosphere during this stage is smaller than the thermal velocity in the surrounding gas.

If the propeller action were the only source of heating of the atmospheric plasma, the magnetospheric boundary of the neutron star would be able to switch its state from subsonic propeller to accretor as its spin period reaches $P_{\mathrm{br}}$. However, as shown below, an additional heating of the atmospheric plasma occurs due to a radial plasma drift through the atmosphere. This additional heating mechanism turns out to be not effective if a star is situated in a relatively strong stellar wind, but in the opposite case it must be taken into account.

\section{Diffusion-driven accretion}

Although the interchange instabilities of the magnetospheric boundary during the subsonic propeller state are suppressed, the "magnetic gates" are not closed completely: the atmospheric plasma is able to penetrate into the stellar magnetic field due to diffusion. The diffusion rate is limited to (see e.g. Ikhsanov 2001b)

$\dot{M}_{\mathrm{B}} \lesssim 10^{11} \mathrm{~g} \mathrm{~s}^{-1} \zeta_{0.1}^{1 / 2} \mu_{30}^{-1 / 14} m^{1 / 7}\left(\frac{\dot{M}_{\mathrm{c}}}{10^{15} \mathrm{~g} \mathrm{~s}^{-1}}\right)^{11 / 14}$.

Here $\zeta_{0.1}=\zeta / 0.1$ is the diffusion efficiency, which is normalized following the results of experiments on the nuclear fusion (e.g. Hamasaki et al. 1974) and the measurements of the solar wind penetrating the magnetosphere of the Earth (Gosling et al. 1991).

This means that the hot atmosphere surrounding the magnetosphere of the star in the subsonic propeller state cannot be purely stationary. For the atmosphere to remain in an equilibrium state, the amount of material flowing from its base into the magnetosphere must be compensated for by the same amount of material coming into the atmosphere through its outer boundary. Thus the radial drift of plasma through the atmosphere with the rate $\dot{M}_{\mathrm{B}}$ and the velocity

$V_{\mathrm{dr}}=\left(\dot{M}_{\mathrm{B}} / \dot{M}_{\mathrm{c}}\right) V_{\mathrm{ff}}$

towards the neutron star is expected.

The structure of the atmosphere with the radial plasma drift can be explain in terms of the quasi-stationary model of Davies \& Pringle (1981) as long as the characteristic time of the accretion process,

$t_{\mathrm{dr}}=R / V_{\mathrm{dr}}$,

is larger than the characteristic time of turbulent motions $t_{\mathrm{t}}=R / V_{\mathrm{t}}$. According to Davies \& Pringle (1981), the velocity of turbulence motions can be approximated as $V_{\mathrm{t}} \simeq$ $\left(R_{\mathrm{m}} / R\right)^{1 / 6} \omega R_{\mathrm{m}}$. Therefore, the condition $t_{\mathrm{t}} \ll t_{\mathrm{dr}}$ proves to be satisfied if the spin period of the star is $P_{\mathrm{s}} \ll P_{\mathrm{qs}}$, where

$P_{\mathrm{qs}, \mathrm{B}} \simeq 1.7 \times 10^{5} \zeta_{0.1}^{-1 / 2} \dot{M}_{15}^{-3 / 14} m^{-6 / 7} \mu_{30}^{13 / 14} \mathrm{~s}$.

Thus, as long as the spin period of the star is $P_{\mathrm{cd}} \lesssim P_{\mathrm{s}} \ll$ $P_{\mathrm{qs}, \mathrm{B}}$, the magnetosphere of the star is surrounded by a hot
( $\left.T \simeq T_{\mathrm{ff}}\right)$ atmosphere in which the plasma pressure is $p \propto$ $R^{-5 / 2}$, the sound speed is $c_{\mathrm{S}} \propto R^{-1 / 2}$ and the number density is $n \propto R^{-3 / 2}$ (for discussion see Davies \& Pringle 1981).

The radial drift of plasma through the atmosphere towards the neutron star leads to the release of the accretion (potential) energy, which is mainly spent in heating the atmospheric plasma. The heating rate due to this process is $L_{\mathrm{dr}}(R) \simeq$ $\dot{M}_{\mathrm{B}} G M_{\mathrm{ns}} / R$. This value is small in comparison with that of the spin-down power (see Davies \& Pringle 1981, Eq. (3.2.4)),

$L_{\mathrm{ssp}}=1.2 \times 10^{36} \mu_{30}^{2} \mathrm{~m}^{-1} P_{\mathrm{s}}^{-3} \mathrm{erg} \mathrm{s}^{-1}$,

as long as the spin period of the star is $P_{\mathrm{s}} \lesssim P_{\mathrm{acc}, \mathrm{B}}$, where

$P_{\mathrm{acc}, \mathrm{B}} \simeq 450 \zeta_{0.1}^{1 / 6} \mu_{30}^{37 / 42} \dot{M}_{15}^{-5 / 14} m^{-16 / 21} \mathrm{~s}$.

However, under the condition $P_{\mathrm{s}}>P_{\text {acc }}$ the heating of the atmospheric plasma is governed by the accretion power.

The characteristic time of the heating due to accretion process is $t_{\mathrm{dr}}$. On the other hand, the cooling time of the atmospheric plasma due to the bremsstrahlung emission is

$t_{\mathrm{cool}} \approx t_{\mathrm{br}} \simeq 633 \mathrm{~s}\left(\frac{T}{10^{9} \mathrm{~K}}\right)^{1 / 2}\left(\frac{n}{10^{13} \mathrm{~cm}^{-3}}\right)^{-1}$,

where $n$ is the number density of the atmospheric plasma, which can be expressed as $n(R)=n\left(R_{\mathrm{m}}\right)\left(R_{\mathrm{m}} / R\right)^{3 / 2}$, where

$n\left(R_{\mathrm{m}}\right)=\frac{\mu^{2}}{4 \pi R_{\mathrm{m}}^{6} k T\left(R_{\mathrm{m}}\right)}$.

Hence, for the temperature of the atmospheric plasma to be smaller than the critical value, $T_{\mathrm{cr}}$, the following condition should be satisfied

$t_{\mathrm{cool}}\left(R_{\alpha}\right)<t_{\mathrm{dr}}\left(R_{\alpha}\right)$

I require this condition to be satisfied at the outer boundary of the atmosphere since $t_{\mathrm{c}} \propto R$ and $t_{\mathrm{dr}} \propto R^{3 / 2}$. Combining Eqs. (11), (12), (16), and (17), one finds that the bremsstrahlung cooling dominates the heating due to accretion power only if the strength of the stellar wind is $\dot{M}_{\mathrm{c}} \gtrsim \dot{M}_{0}$, where

$\dot{M}_{0} \simeq 3 \times 10^{14} \zeta_{0.1}^{7 / 17} \mu_{30}^{-1 / 17} m^{16 / 17} V_{8}^{14 / 17} \mathrm{~g} \mathrm{~s}^{-1}$

This means that a magnetized isolated neutron star is able to switch its state from subsonic propeller to steady accretor only if the mass capture rate by this star from the interstellar medium is $\dot{M}_{\mathrm{c}} \gtrsim \dot{M}_{0}$. Otherwise, the corresponding state transition does not occur and the star remains surrounded by the hot atmosphere. In this case the mass accretion rate onto the stellar surface is limited to $\dot{M} \lesssim \dot{M}_{\mathrm{B}}$ (see Eq. (10)) and, correspondingly, the accretion luminosity is $L_{\mathrm{x}} \lesssim L_{\mathrm{max}}$, where

$L_{\max } \simeq 10^{30} \zeta_{0.1}^{1 / 2} \mu_{30}^{-1 / 14} m^{8 / 7} R_{6}^{-1} \dot{M}_{14}^{11 / 14} \mathrm{erg} \mathrm{s}^{-1}$

Here $R_{6}$ is the radius of the neutron star expressed in units of $10^{6} \mathrm{~cm}$, and $\dot{M}_{14}=\dot{M}_{\mathrm{c}} / 10^{14} \mathrm{~g} \mathrm{~s}^{-1}$. 


\section{Discussion}

Application of our findings to the case of INSs leads to the following conclusions. First, comparing Eqs. (4) and (19) one finds that under the conditions of interest the maximum possible strength of the stellar wind of an isolated neutron star, $\dot{M}_{\text {max }}$, is smaller (at least by a factor of 3) than $\dot{M}_{0}$. Therefore, the interchange instabilities of the magnetospheric boundary of these stars are suppressed and the plasma penetration from the base of the hot atmosphere into the stellar magnetic field is governed by the Bohm diffusion. In this case the accretion luminosity of INSs, whose age is smaller than the characteristic time of the magnetic field decay, $t_{\mathrm{mfd}}$, is limited to

$$
\begin{aligned}
L_{\mathrm{x}}\left(t<t_{\mathrm{mfd}}\right)= & \dot{M}_{\mathrm{B}} \frac{G M_{\mathrm{ns}}}{R_{\mathrm{ns}}} \simeq 3 \times 10^{28} \mathrm{erg} \mathrm{s}^{-1} \\
& \times \zeta_{0.1}^{1 / 2} \mu_{30}^{-1 / 14} m^{8 / 7} R_{6}^{-1}\left(\frac{\dot{M}_{\mathrm{c}}}{10^{12} \mathrm{~g} \mathrm{~s}^{-1}}\right)^{11 / 14} .
\end{aligned}
$$

This radiation is to be emitted from local regions situated at the magnetic poles of the star. That is why the star could be observed as a low luminous pulsing X-ray source. The minimum period of this pulsar is (see Eq. (6))

$P_{\min } \simeq 450 \mu_{30}^{6 / 7} \mathrm{~m}^{-5 / 7} \dot{M}_{12}^{-3 / 7} \mathrm{~s}$.

Second, as the age of an INS becomes comparable with $t_{\mathrm{mfd}}$, the magnetic field is almost unable to control the accretion flow in the stellar vicinity and the direct accretion onto the stellar surface occurs. The accretion luminosity of the star in this case is $L_{\mathrm{x}}=\dot{M}_{\mathrm{c}} G M_{\mathrm{ns}} / R_{\mathrm{ns}}$, i.e. by a factor of $\dot{M}_{\mathrm{c}} / \dot{M}_{\mathrm{B}}$ larger than the accretion luminosity of a neutron star in the state of the subsonic propeller. This radiation is to be emitted from the whole surface of the star and, therefore, the pulsations are not expected to be observed.

Finally, for $\dot{M}_{\mathrm{c}} \lesssim 10^{12} \mathrm{~g} \mathrm{~s}^{-1}$, the spin-down time scale of a neutron star in the subsonic propeller state is (see Davies \& Pringle 1981, Eq. (3.2.5))

$t_{\text {acc, } \mathrm{B}} \gtrsim 10^{9} \zeta_{0.1}^{-1 / 2} m^{1 / 7} \mu_{30}^{-15 / 14} \dot{M}_{12}^{-3 / 14} P_{6} \mathrm{yr}$, where $P_{6}=P_{\text {acc, } \mathrm{B}} / 10^{6}$ s (see Eq. (15)). This time scale is comparable with the characteristic time scale of the magnetic field decay (see e.g. Urpin et al. 1996). Hence, the hot atmosphere surrounding the neutron star in the propeller state can be treated within the quasi-static approximation suggested by Davies \& Pringle (1981).

Acknowledgements. I would like to thank the referee, Dr. Marina Romanova, for useful comments and suggested improvements. I acknowledge the support of the Alexander von Humboldt Foundation within the Long-term Cooperation Program.

\section{References}

Arons, J., \& Lea, S. M. 1976, ApJ, 207, 914

Davies, R. E., Fabian, A. C., \& Pringle, J. E. 1979, MNRAS, 186, 779

Davies, R. E., \& Pringle, J. E. 1981, MNRAS, 196, 209

Elsner, R. F., \& Lamb, F. K. 1976, Nature, 262, 356

Gosling, J. T., Thomsen, M. F., Bame, S. J., et al. 1991, J. Geophys. Res., 96, 14097

Hamasaki, S., Davidson, R. C., Krall, N. A., \& Liewer, P. C. 1974, Nucl. Fusion, 14, 27

Ikhsanov, N. R. 2001a, A\&A, 368, L5

Ikhsanov, N. R. 2001b, A\&A, 367, 549

Ikhsanov, N. R. 2001c, A\&A, 374, 1030

Ikhsanov, N.R. 2002, A\&A, 381, L61

Popov, S. B., Colpi, M., Prokhorov, A., et al. 2000a, ApJ, 544, L53

Popov, S. B., Colpi, M., Treves, A., et al. 2000b, ApJ, 530, 896

Romanova, M. M., Toropina, O. D., Toropin, Yu. M., \& Lovelace, R. V. E. 2002 [astro-ph/0209548]

Shvartsman, V. F. 1970, Radiofizika, 13, 1852

Toropin, Yu. M., Toropina, O. D., Savelyev, V. V., et al. 1999, ApJ, 517, 906

Toropina, O. D., Romanova, M. M., Toropin, Yu. M., \& Lovelace, R. V. E. 2001, ApJ, 561, 964

Treves, A., \& Colpi, M. 1991, A\&A, 247, 107

Treves, A., Turolla, R., Zane, S., \& Colpi, M. 2000, PASP, 112, 297

Urpin, V., Geppert, U., \& Konenkov, D. Yu. 1996, A\&A, 307, 807 